\title{
Quantum phase transition induced by Dzyaloshinskii-Moriya interactions in the kagome antiferromagnet
}

\author{
O. Cépas, ${ }^{1}$ C. M. Fong, ${ }^{2}$ P. W. Leung, ${ }^{2}$ and C. Lhuillier ${ }^{1}$ \\ ${ }^{1}$ Laboratoire de Physique Théorique de la Matière Condensée, UMR7600 CNRS, Université Pierre-et-Marie-Curie, \\ Paris 6, 75252 Paris Cedex 05, France \\ ${ }^{2}$ Department of Physics, Hong Kong University of Science and Technology, Clear Water Bay, Hong Kong
}

(Received 2 September 2008; published 23 October 2008)

\begin{abstract}
We argue that the $S=1 / 2$ kagome antiferromagnet undergoes a quantum phase transition when the Dzyaloshinskii-Moriya coupling is increased. For $D<D_{c}$ the system is in a moment-free phase, and for $D$ $>D_{c}$ the system develops antiferromagnetic long-range order. The quantum critical point is found to be $D_{c}$ $\simeq 0.1 \mathrm{~J}$ using exact diagonalizations and finite-size scaling. This suggests that the kagome compound $\mathrm{ZnCu}_{3}(\mathrm{OH})_{6} \mathrm{Cl}_{3}$ may be in a quantum critical region controlled by this fixed point.
\end{abstract}

DOI: 10.1103/PhysRevB.78.140405

PACS number(s): 75.10.Jm, 75.40.-s

In the search for materials realizing a spin-liquid ground state, one has to face the presence of small anisotropic interactions of spin-orbit origin. Such interactions that break the full rotation symmetry of the Heisenberg model, reduce the quantum fluctuations, and may tend to induce magnetic phases at low temperatures. The recently discovered spin $1 / 2$ copper oxide $\mathrm{ZnCu}_{3}(\mathrm{OH})_{6} \mathrm{Cl}_{3}$ (Ref. 1) that has the geometry of a kagome lattice may be a good candidate for a spinliquid. ${ }^{2}$ Experimentally no apparent freezing of the magnetic moments has been found down to very low temperatures ${ }^{3-5}$ despite strong Heisenberg interactions. Exact diagonalizations of the Heisenberg model predict indeed a nonmagnetic state with no magnetic moment. ${ }^{6,7}$ However, smaller interactions of spin-orbit origin are certainly present. In particular those of Dzyaloshinskii-Moriya symmetry $^{8}$ are expected when the magnetic bonds have no inversion center, which is the case of $\mathrm{ZnCu}_{3}(\mathrm{OH})_{6} \mathrm{Cl}_{3}$. ${ }^{1}$ An immediate question is to what extent they affect the nonmagnetic phase.

Dzyaloshinskii-Moriya interactions have been invoked ${ }^{9}$ in the context of $\mathrm{ZnCu}_{3}(\mathrm{OH})_{6} \mathrm{Cl}_{3}$ to explain the enhancement of the spin susceptibility at low temperatures. ${ }^{5}$ NMR measurements of the local susceptibility have provided a different interpretation in terms of the presence of defects in the structure, ${ }^{10}$ a result consistent with a direct fit of the susceptibility, ${ }^{11}$ and corroborated by theoretical calculations. ${ }^{12} \mathrm{~A}$ more direct evidence of anisotropy has been obtained by paramagnetic resonance. ${ }^{13}$ A Dzyaloshinskii-Moriya coupling of order $0.08 \mathrm{~J}$ was needed to explain the linewidth, ${ }^{13}$ which is typical of cuprates. It is therefore a relatively small correction but may be of crucial importance in highly frustrated systems. In fact it is known that an infinitesimally small Dzyaloshinskii-Moriya interaction in the classical kagome favors long-range Néel order with a $\mathbf{Q}=0$ propagation vector and $120^{\circ}$ orientation of the spins. ${ }^{14}$ Spin-wave corrections renormalize down the magnetic moment, but do not suppress it for $D=0.1 \mathrm{~J} .{ }^{15}$ So it was unclear how this Néel phase could be reconciled with experimental observations.

In this Rapid Communication, we show that the proper inclusion of quantum fluctuations leads to a phase transition from a Néel state to a moment-free phase at a quantum critical point that we estimate to be $D_{c} \simeq 0.1 J$. This therefore resolves the contradiction for $\mathrm{ZnCu}_{3}(\mathrm{OH})_{6} \mathrm{Cl}_{3}$ and, further- more, suggests that its magnetic properties may be strongly influenced by the proximity of the quantum critical point. In particular power-law scalings were observed in the dynamical susceptibility ${ }^{5}$ and NMR relaxation times ${ }^{10,16}$ and have been interpreted so far in terms of critical spin-liquid states for the kagome. ${ }^{17,18}$ The present results indicate that the power laws of $\mathrm{ZnCu}_{3}(\mathrm{OH})_{6} \mathrm{Cl}_{3}$ may well originate in the present fixed point. In order to identify the phases in an unbiased way and locate $D_{c}$, we have performed exact diagonalizations of small clusters. At $D=0.1 \mathrm{~J}$, there is a clear emerging low-energy tower of states that collapses onto the ground state such as $1 / N$, thus signaling a broken-symmetry phase. We have calculated the Néel order parameters as function of $D / J$ and have found that they vanish for $D \leqq 0.1 J$, thus leading to a moment-free phase and $D_{c} \simeq 0.1 J$.

The model we are considering is based on the symmetries of the two-dimensional kagome lattice (Fig. 1), ${ }^{14}$

$$
H=\sum_{n n}\left[J \mathbf{S}_{i} \cdot \mathbf{S}_{j}+\mathbf{D}_{i j} \cdot\left(\mathbf{S}_{i} \times \mathbf{S}_{j}\right)\right],
$$

where $n n$ stands for nearest neighbors and $\mathbf{S}_{i}$ is a $S=1 / 2$ quantum spin on site $i$. Thanks to the lack of inversion symmetry at the middle of each bond, interactions of Dzyaloshinskii-Moriya symmetry are allowed between nearest neighbors. According to Moriya's rules, ${ }^{8}$ there are components of $\mathbf{D}_{i j}$ perpendicular to the planes of strength $D$ stag-

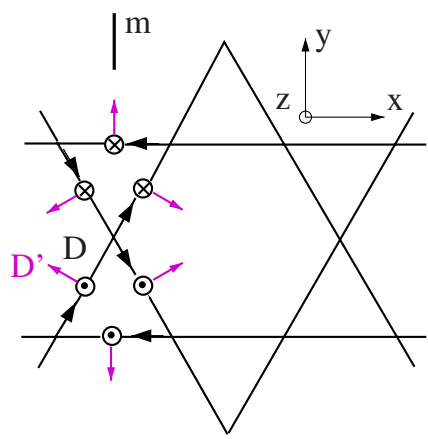

FIG. 1. (Color online) The kagome lattice with the allowed Dzyaloshinskii-Moriya interactions. The orientations of the bonds specify the order of the operators in $\mathbf{S}_{i} \times \mathbf{S}_{j} . m$ is a mirror plane. 


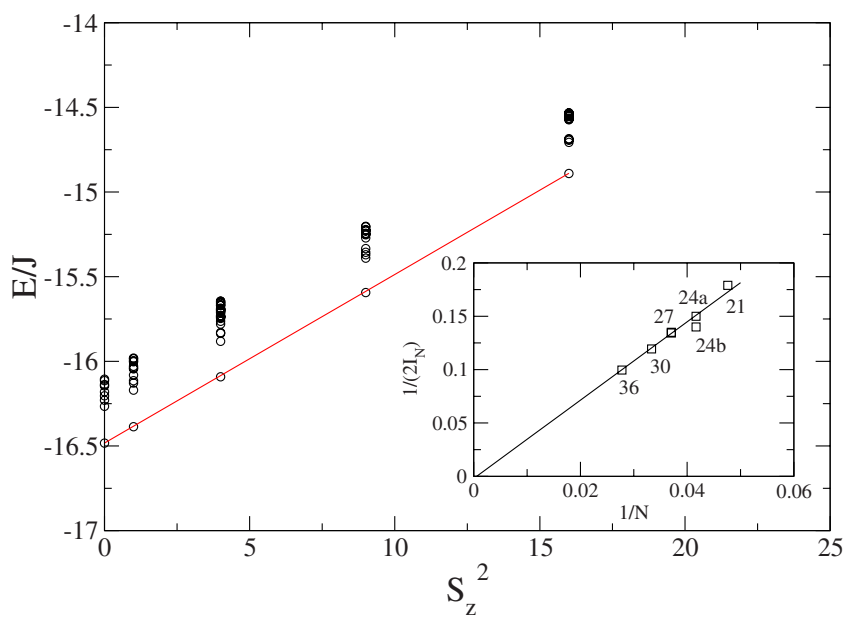

FIG. 2. (Color online) Low-energy levels of the Heisenberg kagome lattice with Dzyaloshinskii-Moriya interactions for $N=36$. The inset gives the slope of the lowest-energy states versus $S_{z}^{2}$, denoted by $1 /\left(2 I_{N}\right)$, as a function of $1 / N$. $(D=0.1 J$. $)$

gered from up triangles to down triangles and in-plane components that point toward the center of each triangle of strength $D^{\prime}$ (see Fig. 1). ${ }^{14}$ There is a priori no simple relation between $D$ and $D^{\prime}$. It is true that if the $\mathrm{Cu}-(\mathrm{OH})-\mathrm{Cu}$ plane was a mirror plane of the crystal structure, then the $\mathbf{D}$ vector would be perpendicular to it. In fact the proton of $(\mathrm{OH})$ breaks that symmetry, ${ }^{1}$ and since the perturbation is expected to be strong it is difficult to relate $D$ and $D^{\prime}$. For $\mathrm{ZnCu}_{3}(\mathrm{OH})_{6} \mathrm{Cl}_{3}, J$ has been estimated from the susceptibility to be about $170-190 \mathrm{~K} \cdot{ }^{9,11}$ Interplane couplings are thought to be smaller because copper ions are far away in the $c$ direction and separated by sheets of zinc and will be neglected in the following. According to electron-spinresonance measurements, $D=15 \mathrm{~K}(D \sim 0.08 J)$ and $D^{\prime}$ $=2 \mathrm{~K}\left(D^{\prime} \sim 0.01 J\right) .{ }^{13}$

We now map the model Eq. (1) onto a simpler model that restores a $\mathrm{U}(1)$ symmetry up to terms of order $D^{\prime 2} / J$. The in-plane components vectors sum up to zero when going around a triangle and are therefore reducible to $D^{\prime 2} / J$ term (for small $\left.D^{\prime}\right)^{19}$ by appropriate rotations of the spin operators. ${ }^{20}$ We shall neglect these second-order terms not only because they are smaller but also because there are other (symmetric) exchange anisotropies at the same order that we have not included. Therefore in the rotated frame, the model has only the original $D$ component along $z$ with the same strength and has a $\mathrm{U}(1)$ rotation symmetry about this axis: it is this symmetry that, as we shall show, is going to be spontaneously broken. In the following we shall present the results of the numerical diagonalization of Eq. (1) for systems of size $N=21,24,27,30,36$ (for the $N=24-27$, we have considered two different cluster shapes). The dimension of the largest Hilbert space is $\sim 7 \times 10^{8}$. We have started with a Dzyaloshinskii-Moriya coupling strength fixed to $D=0.1 \mathrm{~J}$ (and $J=1$ ).

Symmetry breaking in the thermodynamic limit. We have calculated several low-energy levels of Eq. (1) in each sector of the total magnetization, denoted by $S_{z}$, for different cluster sizes. For $N=36$, the energy levels are shown in Fig. 2. The spectrum is qualitatively different from the exact spectra ob- tained at $D=0$ (see Ref. 7). Here we clearly see a band of low-energy levels (red line in Fig. 2) well separated from higher-energy states. It forms a so-called tower of states which energy is very well described by a quadratic term $S_{z}^{2} / 2 I_{N}$ (Fig. 2). The slope $1 /\left(2 I_{N}\right)$ is fitted for all available $N$ and shown in the inset of Fig. 2. It clearly extrapolates to zero in the thermodynamic limit such as $1 / N$. In this limit one can then form a superposition of these eigenstates with different $S_{z}$, thus forming a macroscopic state with a preferred in-plane direction. This therefore shows that the system breaks the rotation symmetry in the thermodynamic limit. In this standpoint, the degeneracy of the tower of states is then a natural consequence of the invariance of the Hamiltonian in rotations about the internal axis defined by $\mathbf{D}$ : a given macroscopic state pointing in the $\mathbf{u}$ direction in the transverse plane is degenerate with all its transforms in any rotation about $\mathbf{D}$. In addition, the wave vector of the lowestenergy state in each $S_{z}$ sector is $\mathbf{Q}=0$ for all clusters so that we can safely conclude that the system will not break translation invariance in the thermodynamic limit. Furthermore, because of the continuous broken symmetry, we expect a long-wavelength Goldstone mode with energy varying such as $k \propto N^{-1 / 2}$ in two dimensions. Unfortunately, the first allowed $k \neq 0$ wave vectors are not small enough on these clusters to observe the individual long-wavelength states directly in the spectra. In fact, indirect confirmation of a Goldstone mode will be given below in the scalings of the energy of the ground state and correlations. From the spectra, we can now extract the uniform susceptibility at $T=0$ by using the expression of the energy, $S_{z}^{2} / 2 I_{N}-H S_{z}$. The susceptibility of the ground state $I_{N}$ is proportional to $N$ (as shown in Fig. 2) and the susceptibility per site $\chi=I_{N} / N$ is found to be $\chi$ $=0.144 \pm 0.002$ for $D / J=0.1$ in the thermodynamic limit.

Néel order parameters. To test for the possibility to have a sublattice magnetization (or Néel order) in the thermodynamic limit, we have calculated the spin-spin correlations in the (finite size) ground state. We distinguish between correlations in a plane perpendicular to $\mathbf{D},\left\langle 0\left|S_{i a}^{x} S_{j b}^{x}+S_{i a}^{y} S_{j b}^{y}\right| 0\right\rangle$ (where $i$ is the unit cell and $a$ the sublattice index), and the correlations along $\mathbf{D},\left\langle 0\left|S_{i a}^{z} S_{j b}^{z}\right| 0\right\rangle$. As expected from the easyplane character of the Dzyaloshinskii-Moriya interaction, the latter remains much smaller and especially at large separation. To study the in-plane ordering, we define the Fourier transform of the spin-spin correlations,

$$
S_{a b}(\mathbf{Q})=\frac{24}{N^{2}} \sum_{i j} e^{i \mathbf{Q} \cdot\left(\mathbf{R}_{i}-\mathbf{R}_{j}\right)}\left\langle 0\left|S_{i a}^{x} S_{j b}^{x}\right| 0\right\rangle .
$$

This is a $3 \times 3$ matrix that turns out to be peaked at $\mathbf{Q}=0$. The largest eigenvalue at $\mathbf{Q}=0$ corresponds simply to the $120^{\circ}$ in-plane orientation of the magnetic moments within the unit cell. The prefactor 24 is chosen so that the largest eigenvalue is 1 in the perfect Néel state. For a quantum magnet with a finite Néel order parameter, the eigenvalue should extrapolate to a finite value smaller than 1 (because of quantum fluctuations), with a well-defined finite-size scaling. The latter can be predicted from the existence of a low-energy spin wave with wave vector varying such as $N^{-1 / 2}$ in two dimensions and is of the form $N^{-1 / 2}$ for the correlations and 


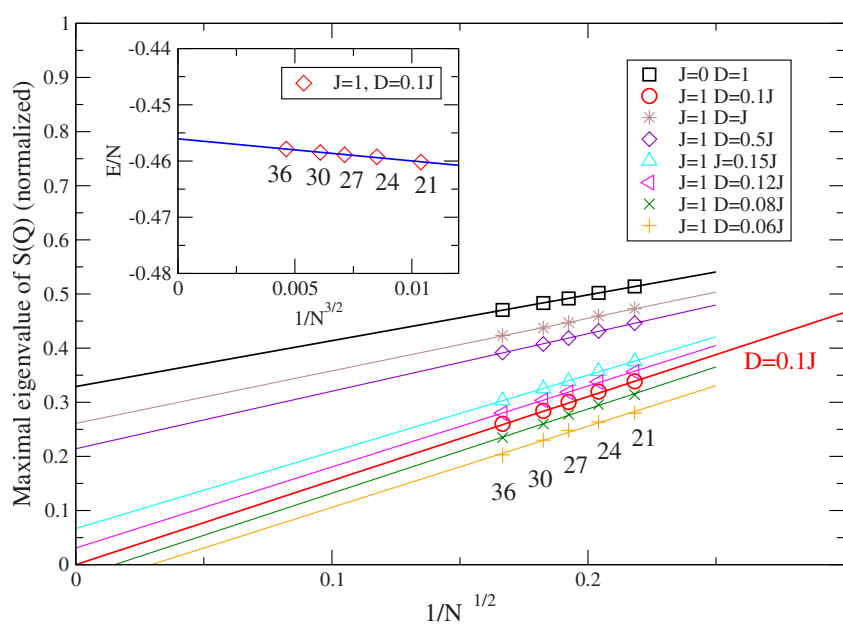

FIG. 3. (Color online) Finite-size extrapolation of the largest eigenvalue of $S_{a b}(\mathbf{Q}=0)$ showing (i) a scaling in $N^{-1 / 2}$; (ii) the decrease in the sublattice moment from $m_{\mathrm{AF}}^{2}=0.326(D=1, J=0)$ to zero $(D=0.1 J)$ and negative values $(D=0.06,0.08 J)$. Inset: finitesize scaling of the ground-state energy in $N^{-3 / 2}$ for $D=0.1 J$.

$N^{-3 / 2}$ for the energy per site. ${ }^{21}$ Figure 3 shows the largest eigenvalue versus $N^{-1 / 2}$ for different $D / J$. First we see that the scaling is obeyed both for the correlations (Fig. 3) and for the ground-state energy per site (inset of Fig. 3). Second, the intercept for infinite system-size gives the order parameter, noted as $m_{\mathrm{AF}}^{2}$. Starting from large $D / J$ (squares) and reducing $D / J$ we see that the order parameter decreases from $m_{\mathrm{AF}}^{2} \sim 0.326$ to zero at $D_{c} \simeq 0.1 J$ (circles). For $D<D_{c}$, it is no longer possible to find a finite order parameter: the negative extrapolated values reflect the breakdown of the scaling at some length-scale and short-range correlations develop instead. In Fig. 4, we summarize the behavior of the Néel order-parameter $m_{\mathrm{AF}}$ as a function of $D / J$. The order parameter decreases continuously to zero so that the transition is compatible with a second-order phase transition. It seems difficult though to extract an accurate value for the critical exponent given the error bars resulting from finite-size scaling. The present data are compatible with the mean-field behavior, $m_{\mathrm{AF}}^{2} \propto\left(D-D_{c}\right)$, but the exponent could also be

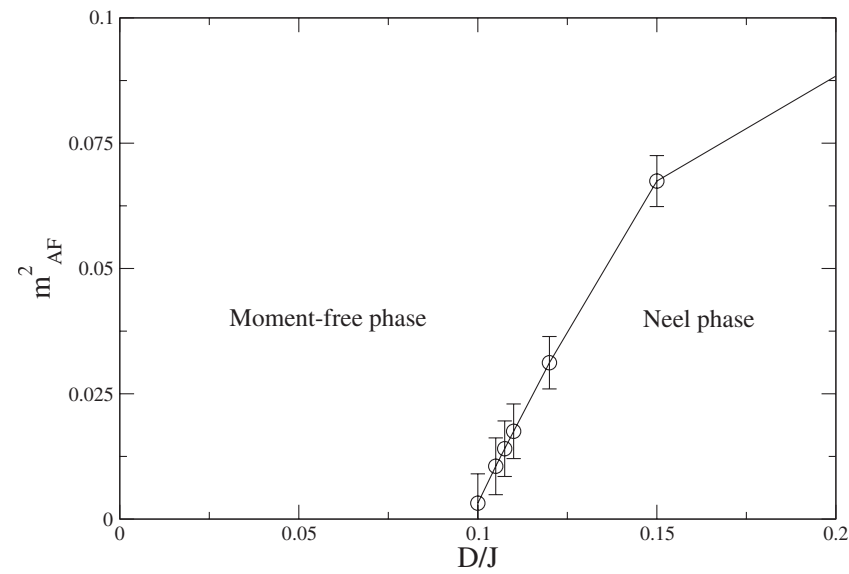

FIG. 4. The Néel order parameter as a function of $D / J$ obtained from extrapolations to infinite size. The system has no magnetic moment for $D<D_{c} \simeq 0.1 J$ and has Néel order for $D>D_{c}$. smaller. In any case, $D_{c} \simeq 0.1 J$ clearly appears as the critical point separating the Néel phase $\left(Q=0,120^{\circ}\right.$ in-plane orientation of the spins $)^{22}$ from a phase with no static moment. It is a more accurate estimate than that of spin waves (that is three times smaller $)^{15}$ because here all quantum fluctuations have been taken into account.

In order to have a simple understanding for the occurrence of a Néel phase in the phase diagram, we discuss the limit of large $D / J$, for which simple physical arguments can be used. For this we start with a trimerized version of the kagome lattice. ${ }^{23}$ On a triangle, three Heisenberg quantum spins $1 / 2$ form a quartet and two degenerate low-energy doublets. The Dzyaloshinskii-Moriya interaction lifts the degeneracy and selects the doublet with the (vector)-chirality opposite to $\mathbf{D}$. We now assume that $D$ is large and ignore the higher doublet (and quartet). We define a pseudospin $\sigma_{i}$ $=1 / 2$ for the lowest doublet on each triangle. The intertriangle Dzyaloshinskii-Moriya interaction (that is now supposed to be large) gives an effective interaction for the pseudospins that we can write as

$$
H^{\prime}=-\frac{2}{9}|D| \sum_{\langle i, j\rangle} \sqrt{3}\left(\sigma_{i}^{x} \sigma_{j}^{x}+\sigma_{i}^{y} \sigma_{j}^{y}\right)+\hat{\mathbf{e}}_{i j} \cdot\left(\sigma_{i} \times \sigma_{j}\right),
$$

where $i, j$ are now sites on a triangular lattice. $\hat{\mathbf{e}}_{i j}$ is a unit vector along $+z$ when turning anticlockwise around an up triangle of the triangular lattice. Equation (3) is essentially a ferromagnetic in-plane interaction with a sizable effective Dzyaloshinskii-Moriya interaction. The latter gives magnetic frustration and the problem of solving Eq. (3) is in principle as complicated as the original problem. For three triangles, the ground state of Eq. (3) is the $\mathbf{Q}=0$ doublet state (of pseudospins) made of $(|\uparrow \uparrow \downarrow\rangle+|\uparrow \downarrow \uparrow\rangle+|\downarrow \uparrow \uparrow\rangle) / \sqrt{3}$ and its time reversal counterpart. Similarly, the classical ground state of Eq. (3) is the $\mathbf{Q}=0$ in-plane ferromagnetic state with, e.g., $\left\langle\sigma_{i}^{x}\right\rangle=1 / 2$ on all triangles. In terms of the original spins, this corresponds to long-range Néel order in $\left\langle\mathbf{S}_{i a}\right\rangle$ of the $\mathbf{Q}$ $=0$ and $120^{\circ}$ form. The reduction in the in-plane moment can also be estimated. For the three-triangle ferromagnetic doublet given above, one can form a superposition of the two states that gives a moment along $x$ for instance. The moment is then found to be a fraction $m_{\mathrm{AF}}=4 / 9 \sim 0.44$ of the full moment. This gives a simple explanation for the $\mathbf{Q}=0$ Néel phase found by exact diagonalization in the limit of large $D / J$. Since we know from previous works that the $D=0$ phase is nonmagnetic, 6,7 we naturally expect at least one quantum critical point between the two phases.

We conclude that the $S=1 / 2$ kagome antiferromagnet has a quantum critical point at $D_{c} \simeq 0.1 J$ separating a momentfree phase $\left(D<D_{c}\right)$ from a Néel phase with a sublattice moment $\left(D>D_{c}\right)$ (or a weak net moment if $D^{\prime} \neq 0$, see Ref. 22). This is clearly compatible with the absence of a static moment in $\mathrm{ZnCu}_{3}(\mathrm{OH})_{6} \mathrm{Cl}_{3}$ (Refs. 3-5) because the coupling extracted from ESR, $D=0.08 J,{ }^{13}$ is smaller than the critical coupling. ${ }^{24}$ Furthermore this estimation places this compound very close to the quantum critical point. This raises the issue of the origin of the power-law behaviors observed experimentally when the temperature is decreased. $5,10,16$ They have been interpreted so far in terms of critical spin 
liquids ${ }^{17,18}$ or free $S=1 / 2$ impurities. ${ }^{25}$ Here we suggest a different intrinsic interpretation in terms of the proximity with the present critical point. The prediction of the critical behavior and low-temperature scalings is not an easy task, however. In view of the present uncertainties about the $D$ $=0$ phase and the other possibilities of valence bond crystal states $^{26}$ or intermediate phases such as a spin nematic state, for instance, it is indeed difficult to ascertain what the effective low-energy quantum field theory is. It is interesting to note though that the same ordered phase was found as an instability of the field theory describing the algebraic spin liquid, ${ }^{27}$ but here we have given a finite critical value for $D_{c}$. At finite temperatures such a critical point will open a quan- tum critical region that may have consequences on the magnetic properties of $\mathrm{ZnCu}_{3}(\mathrm{OH})_{6} \mathrm{Cl}_{3}$. It is also of course of particular interest if the compound could be driven across the transition by applying an external pressure or magnetic field, for instance.

We would like to thank F. Bert, P. Mendels, and A. Zorko for sharing with us unpublished experimental data, and B. Canals, C. Lacroix, A. Laeuchli, and F. Mila for discussions. O.C. would like to thank the ILL and the Institut Néel for their hospitality. C.M.F. and P.W.L. were supported by the Hong Kong RGC Grant No. 601207.
${ }^{1}$ M. P. Shores, E. A. Nytko, B. M. Bartlett, and D. G. Nocera, J. Am. Chem. Soc. 127, 13462 (2005).

${ }^{2}$ B. Levi, Phys. Today 60, 16 (2007).

${ }^{3}$ P. Mendels, F. Bert, M. A. de Vries, A. Olariu, A. Harrison, F. Duc, J. C. Trombe, J. S. Lord, A. Amato, and C. Baines, Phys. Rev. Lett. 98, 077204 (2007).

${ }^{4}$ O. Ofer, A. Keren, E. A. Nytko, M. P. Shores, B. M. Bartlett, D. G. Nocera, C. Baines, and A. Amato, arXiv:cond-mat/0610540 (unpublished).

${ }^{5}$ J. S. Helton, K. Matan, M. P. Shores, E. A. Nytko, B. M. Bartlett, Y. Yoshida, Y. Takano, A. Suslov, Y. Qiu, J.-H. Chung, D. G. Nocera, and Y. S. Lee, Phys. Rev. Lett. 98, 107204 (2007).

${ }^{6}$ P. W. Leung and V. Elser, Phys. Rev. B 47, 5459 (1993).

${ }^{7}$ P. Lecheminant, B. Bernu, C. Lhuillier, L. Pierre, and P. Sindzingre, Phys. Rev. B 56, 2521 (1997); C. Waldtmann, H.-U. Everts, B. Bernu, C. Lhuillier, P. Sindzingre, P. Lecheminant, and L. Pierre, Eur. Phys. J. B 2, 501 (1998).

${ }^{8}$ I. Dzyaloshinskii, J. Phys. Chem. Solids 4, 241 (1958); T. Moriya, Phys. Rev. 120, 91 (1960).

${ }^{9}$ M. Rigol and R. R. P. Singh, Phys. Rev. Lett. 98, 207204 (2007); Phys. Rev. B 76, 184403 (2007).

${ }^{10}$ A. Olariu, P. Mendels, F. Bert, F. Duc, J. C. Trombe, M. A. de Vries, and A. Harrison, Phys. Rev. Lett. 100, 087202 (2008).

${ }^{11}$ G. Misguich and P. Sindzingre, Eur. Phys. J. B 59, 305 (2007).

${ }^{12}$ M. J. Rozenberg and R. Chitra, arXiv:0805.3483 (unpublished).

${ }^{13}$ A. Zorko, S. Nellutla, J. van Tol, L. C. Brunel, F. Bert, F. Duc, J. C. Trombe, M. A. de Vries, A. Harrison, and P. Mendels, Phys. Rev. Lett. 101, 026405 (2008).

${ }^{14}$ M. Elhajal, B. Canals, and C. Lacroix, Phys. Rev. B 66, 014422 (2002).

${ }^{15}$ M. Elhajal, Ph.D. thesis, University Joseph Fourier, 2002; R. Ballou, B. Canals, M. Elhajal, C. Lacroix, and A. S. Wills, J. Magn. Magn. Mater. 262, 465 (2003).
${ }^{16}$ T. Imai, E. A. Nytko, B. M. Bartlett, M. P. Shores, and D. G. Nocera, Phys. Rev. Lett. 100, 077203 (2008).

${ }^{17}$ Y. Ran, M. Hermele, P. A. Lee, and X.-G. Wen, Phys. Rev. Lett. 98, 117205 (2007).

${ }^{18}$ S. Ryu, O. I. Motrunich, J. Alicea, and M. P. A. Fisher, Phys. Rev. B 75, 184406 (2007).

${ }^{19}$ Y. F. Cheng, O. Cépas, P. W. Leung, and T. Ziman, Phys. Rev. B 75, 144422 (2007).

${ }^{20}$ L. Shekhtman, O. Entin-Wohlman, and A. Aharony, Phys. Rev. Lett. 69, 836 (1992).

${ }^{21}$ H. Neuberger and T. Ziman, Phys. Rev. B 39, 2608 (1989); D. S. Fisher, ibid. 39, 11783 (1989).

${ }^{22}$ When $D^{\prime} \neq 0$, we have to transform the operators back onto the original frame: the $120^{\circ}$ order transforms onto a weak ferromagnet. In fact the classical energy difference between the weak ferromagnet and the coplanar $120^{\circ}$ state is only of order $D^{\prime 2}$, and one must include second-order exchange anisotropies to actually predict the form of order (Ref. 20).

${ }^{23}$ V. Subrahmanyam, Phys. Rev. B 52, 1133 (1995); F. Mila, Phys. Rev. Lett. 81, 2356 (1998).

${ }^{24}$ Note also that our estimate of the zero-temperature susceptibility at $D=D_{c}, \chi=0.144$ is close to the intrinsic susceptibility measured by NMR, $\chi=0.13$ (Ref. 10).

${ }^{25}$ R. Chitra and M. J. Rozenberg, Phys. Rev. B 77, 052407 (2008).

${ }^{26}$ J. B. Marston and C. Zeng, J. Appl. Phys. 69, 5962 (1991); C. Zeng and V. Elser, Phys. Rev. B 51, 8318 (1995); A. V. Syromyatnikov and S. V. Maleyev, ibid. 66, 132408 (2002); R. Budnik and A. Auerbach, Phys. Rev. Lett. 93, 187205 (2004); P. Nikolic and T. Senthil, Phys. Rev. B 68, 214415 (2003); R. R. P. Singh and D. A. Huse, ibid. 76, 180407(R) (2007).

${ }^{27}$ M. Hermele, Y. Ran, P. A. Lee, and X.-G. Wen, Phys. Rev. B 77, 224413 (2008). 